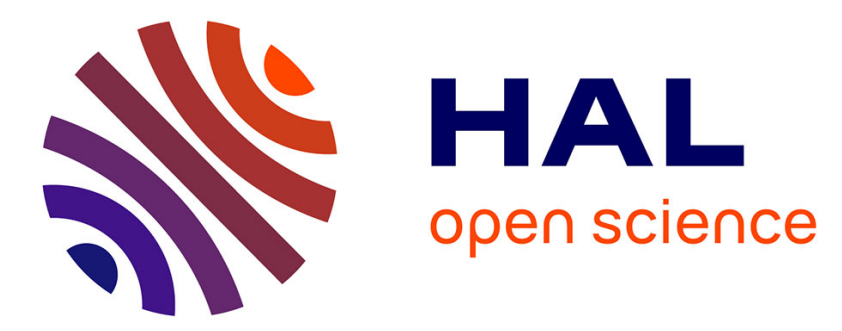

\title{
Un bilan sur la circulation monétaire préaugustéenne à Lattes
}

\author{
Michel Py
}

\section{To cite this version:}

Michel Py. Un bilan sur la circulation monétaire préaugustéenne à Lattes. Gallia - Archéologie de la France antique, 2008, Lattara/Lattes (Hérault): nouveaux acquis, nouvelles questions sur une ville portuaire protohistorique et romaine, 65, pp.169-174. 10.3406/galia.2008.3339 . hal-01913820

\section{HAL Id: hal-01913820 \\ https://hal.science/hal-01913820}

Submitted on 19 Dec 2019

HAL is a multi-disciplinary open access archive for the deposit and dissemination of scientific research documents, whether they are published or not. The documents may come from teaching and research institutions in France or abroad, or from public or private research centers.
L'archive ouverte pluridisciplinaire HAL, est destinée au dépôt et à la diffusion de documents scientifiques de niveau recherche, publiés ou non, émanant des établissements d'enseignement et de recherche français ou étrangers, des laboratoires publics ou privés.

\section{(이) $\$$}

Distributed under a Creative Commons Attribution - NonCommercial - NoDerivatives| 4.0 


\title{
UN BILAN SUR LA CIRCULATION MONÉTAIRE PRÉAUGUSTÉENNE À LATTES
}

\author{
Michel PY
}

Mots-clés. Âge du Fer, numismatique, monnaie, circulation monétaire, économie, troc, échanges.

Résumé. L’analyse récente de près de 6000 monnaies préaugustéennes recueillies dans les fouilles de Lattes, tant dans les premiers sondages d'Henri Prades que dans les fouilles programmées, a fourni l'occasion de préciser les caractéristiques de l'usage de la monnaie sur le site et de replacer ces données dans le cadre des circulations monétaires protohistoriques en Gaule méridionale. De plus, la découverte de quatre trésors de monnaies d'argent, datés entre la fin du IV ${ }^{e}$ s. et le milieu du II ${ }^{e}$ s. av. J.-C., a permis d'aborder la question des relations entre la circulation interne du numéraire, qui ne se développe que fort tard, et la thésaurisation qui apparaît intimement liée sur ce site à l'activité marchande. L'approvisionnement de Lattes en monnaies est dominé durant l'âge du Fer et jusqu'au changement d'ère par les productions massaliètes, toujours majoritaires, voire exclusives aux premières périodes, mais la diversité des autres émissions, notamment méditerranéennes, témoigne à sa manière de l'activité du port.

Key-words. Iron Age, numismatics, coinage, currency circulation, economy, exchange, trade.

Abstract. The recent analysis of nearly 6,000 pre-Augustan coins found during the investigations at Lattes, through the first surveys by Henri Prades as in the programmed excavations, gave the opportunity to define the characteristics in the currency habit on the site and to set these informations in the background of Protohistoric coin circulations in southern Gaul. Besides, the discovery of four silver coin hoards, dated to the end of the $4^{\text {th }}$ century up to mid $3^{\text {rd }}$ century B.C., made it possible to raise the question of relations between local currency circulation, expanding very late and thesaurization which seems connected to trading activities on the site. Coin supplying of Lattes is dominated during the Iron Age until the changing era by Massaliote productions, always being the majority or exclusive to the early periods, but the diversity of other issues, notably Mediterranean, attests in its own way to the port activity.

Translation: Isabelle FAUDUET

Schlüsselwörter. Eisenzeit, Numismatik, Münzen, Geldumlauf, Wirtschaft, Tauschhandel, Handel.

Zusammenfassung. Die vor kurzem durchgeführte Analyse von 6000 voraugusteischen Münzen aus den Grabungen von Lattes, die sowohl aus den ersten Sondierungen von Henri Prades als auch aus den Plangrabungen stammten, bot Gelegenheit den Geldumlauf in Lattes zu untersuchen und die gewonnen Daten in den Rahmen der frühgeschichtlichen Geldzirkulation in Südgallien einzuordnen. Darüber hinaus hat die Entdeckung von vier Depots von Silbermünzen, die aus der Zeit zwischen dem Ende des 4. und der Mitte des 2. Jahrhunderts v.u.Z. datieren, ermöglicht, die Frage zu stellen nach den Beziehungen zwischen der internen Zirkulation der Münzen, die sich extrem spät entwickelt, und der Thesaurierung, die in Lattes eng mit dem Handel verbunden zu sein scheint. Lattes wird während der Eisenzeit und bis zur Zeitenwende überwiegend und in den ersten Phasen sogar ausschließlich mit Münzen aus Marseille versorgt, doch die Vielfalt der anderen Münzen, insbesondere aus dem Mittelmeerraum, zeugt auf ihre Weise von der Aktivität des Hafens.

Übersetzung: Isa ODENHARDT-DONVEZ

L'histoire monétaire de Lattara protohistorique a fait récemment l'objet d'un bilan complet, incluant les découvertes anciennes et récentes, qui a fourni l'occasion d'une révision de la problématique générale de la circulation monétaire en Gaule méridionale avant l'époque d'Auguste (Py, 2006). Il faut rappeler que ce site offre, avec Toulouse, l'un des plus importants médaillers illustrant cette période dans le midi de la France : les recherches préliminaires 

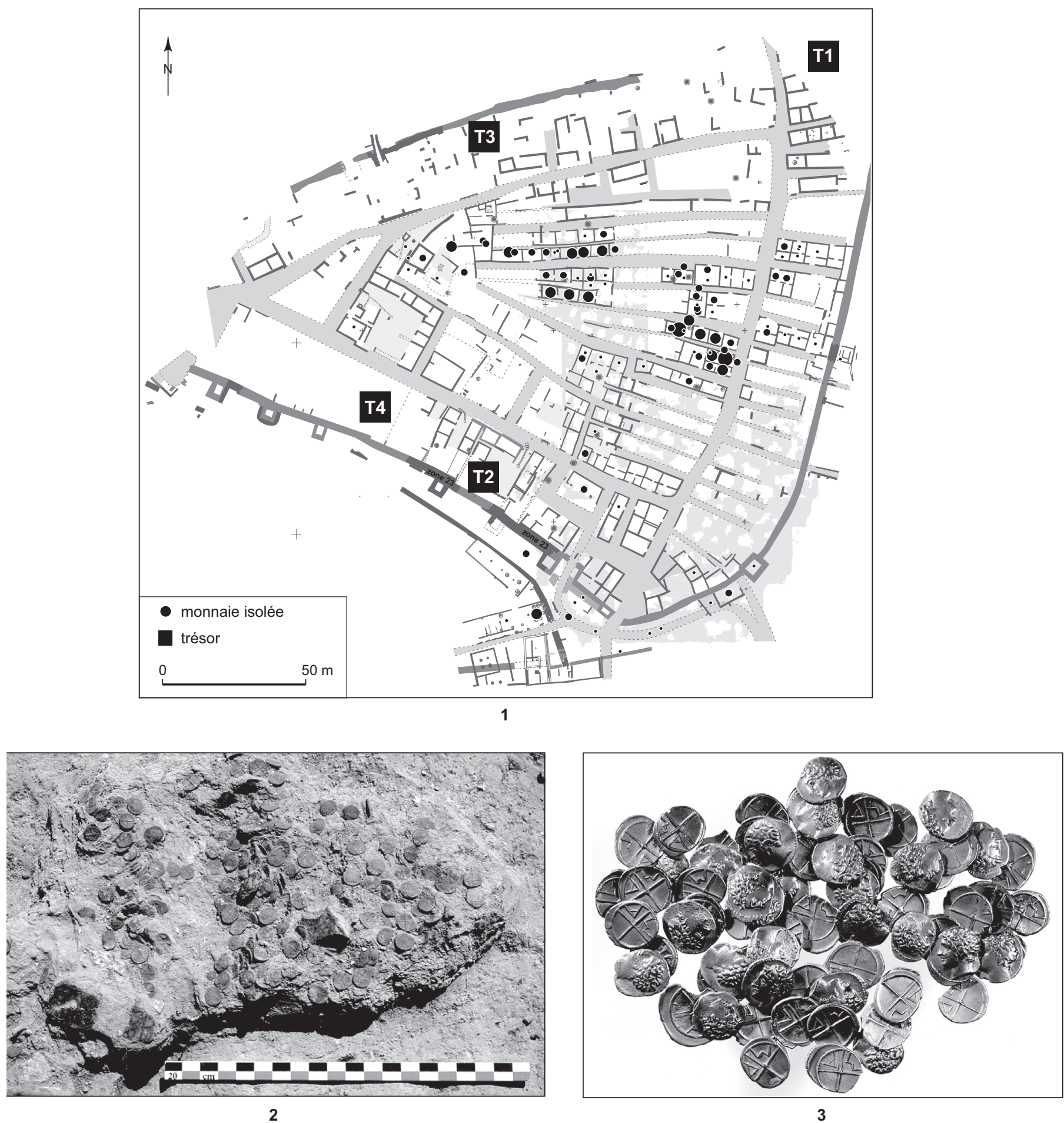

Fig. 114 - Répartition des principales découvertes monétaires et des quatre trésors préromains dans la ville ancienne de Lattara (1) et évocation du trésor d'oboles massaliètes $n^{0} 4$ (fin du III $s$.) découvert durant la campagne de fouille 2000 (2 et 3) (DAO : (C) UFRAL; clichés : M. Py, CNRS). 
menées par le groupe archéologique Painlevé avaient livré 1618 monnaies dispersées dans différents secteurs de l'habitat (dont seules les 531 premières ont été étudiées : Majurel et al., 1976 ; Richard J.-C., 1978), et trois trésors monétaires totalisant au moins 3839 monnaies (Majurel et al., 1966 et 1967). Les fouilles programmées entamées en 1983 ont fourni jusqu'en 2005 quelque 846 monnaies dispersées et un quatrième trésor de 786 oboles, soit un effectif total de 7089 monnaies (fig. 114). Parmi elles, une fois déduits quelques exemplaires perdus de certains trésors et les exemplaires frustes de l'habitat, 5992 monnaies plus ou moins précisément identifiables illustrent les phases préaugustéennes.

Cet ensemble, considérable au plan quantitatif, présente également quelques avantages sur le plan qualitatif par rapport à d'autres collections. D'une part, il a été recueilli sur un site unique et homogène, témoignant d'une occupation particulièrement longue : la période envisagée s'étend en effet sur cinq siècles, de la fin du $\mathrm{VI}^{\mathrm{e}} \mathrm{s}$. à la fin du I $^{\text {er }}$ s. av. J.-C. (même si la monnaie n'est attestée sur le site qu'à partir du milieu du IV ${ }^{\mathrm{e}}$ s., mais les absences sont aussi significatives), offrant l'opportunité d'une analyse à la fois diachronique et statistique approfondie. D'autre part, une proportion notable des monnaies retrouvées dans les couches d'habitat au cours des fouilles programmées est issue d'unités stratigraphiques précisément documentées et bénéficie d'une datation absolue par le contexte. Enfin, la découverte sur le même lieu, dans l'enceinte de la ville primitive de Lattara, de quatre importants trésors monétaires préromains autorise une approche des relations entre circulation et thésaurisation dans un contexte socio-économique de mieux en mieux connu.

Cette agglomération portuaire à laquelle on reconnaît dès l'origine un rôle important dans le développement du commerce régional fonctionne apparemment durant les deux premiers siècles de son histoire sans connaître la monnaie, alors que celle-ci est attestée de part et d'autre, autour de Marseille (Furtwängler, 1978) et dans une moindre mesure sur le littoral du Languedoc occidental. Insistons cependant sur le fait que cette absence reflète l'état actuel des connaissances, et non une donnée définitive : s'il apparaît certain que la monnaie n'est pas utilisée à Lattes entre la fin du VI ${ }^{\mathrm{e}} \mathrm{s}$. et le milieu du $\mathrm{IV}^{\mathrm{e}} \mathrm{s}$. av. J.-C. pour les échanges intercommunautaires qui se déroulent encore totalement selon des mécanismes assimilables au troc, l'argument a silentio n'offre aucune certitude pour ce qui concerne les transactions avec les négociants méditerranéens qui abordent au port, la découverte future de trésors

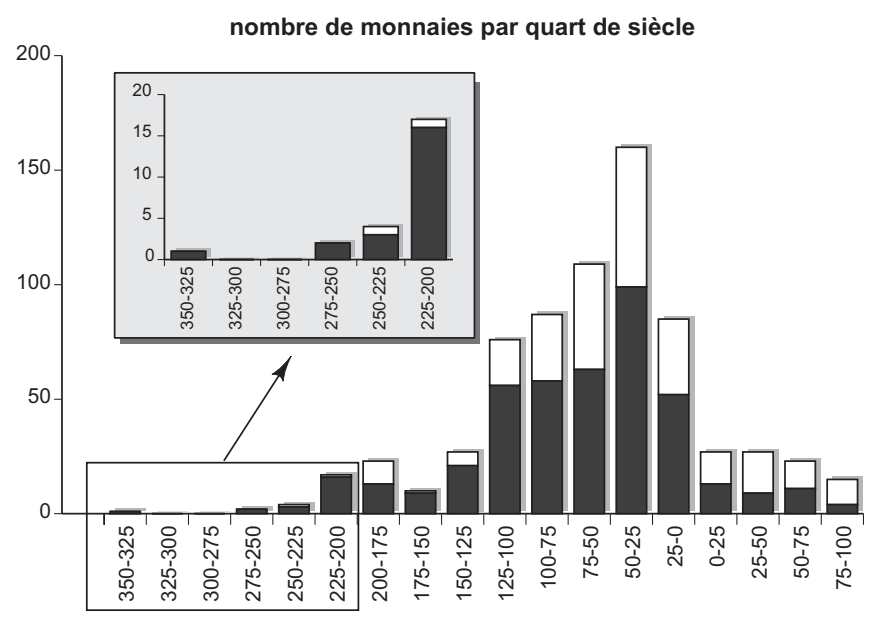

Fig. 115 - Évolution de la circulation monétaire sur le site de Lattes d'après le décompte des monnaies datées par le contexte stratigraphique: en noir, la part de Marseille dans le numéraire (DAO : M. Py, CNRS).

plus anciens que ceux que l'on connaît actuellement n'étant à mon sens nullement exclue.

C'est du moins l'enseignement que l'on peut tirer de l'examen de la phase ultérieure (milieu du $\mathrm{IV}^{\mathrm{e}}-\mathrm{III}{ }^{\mathrm{e}} \mathrm{s}$.), durant laquelle le numéraire demeure extrêmement rare dans l'habitat (quelques pièces tout au plus) (fig. 115), mais où trois dépôts monétaires représentant plusieurs milliers d'oboles de Marseille en argent ont été découverts dans l'enceinte de la ville (fig. 114). Ces trésors, dans lesquels j’ai proposé de voir les restes de capitaux marchands liés principalement au commerce avec les étrangers, appartiennent à une sphère spécifique au sein de laquelle la monétisation des rapports d'échange a pu progresser de manière beaucoup plus précoce que dans la sphère interne. L'unicité d'origine des monnaies attestées jusqu'à la fin du $\mathrm{III}^{\mathrm{e}}$ s., toutes massaliètes, montre clairement que Marseille constitue l'interlocuteur privilégié (pour ne pas dire unique) des Lattarenses, et illustre, comme les importations de vaisselle et d'amphores de même provenance qui dominent outrageusement le marché local, le quasi monopole que la cité phocéenne a mis en place et consolidé dans cette région, après avoir écarté les négociants étrusques installés à Lattes au début du $\mathrm{V}^{\mathrm{e}} \mathrm{s}$.

Un tournant se situe vers la fin du III $^{\mathrm{e}}$ s. av. J.-C., quand apparaissent concurremment les petits et moyens bronzes massaliètes et les premières émissions locales. Ces deux nouveautés portent en germe les caractères de l'évolution ultérieure, faite à la fois de diversification et de développement de l'usage monétaire (fig. 115).

La création de petites dénominations de bronze par Marseille, qui peut être synchronisée avec l'émission des 


\begin{tabular}{|l|c|c|c|c|c|c|c|}
\hline \multicolumn{1}{|c|}{$\mathbf{2 5 0 - 2 2 5}$} & \multicolumn{1}{|c|}{ Dénomination } & Abréviation & Équivalence & Rapport à l'obole & $\begin{array}{c}\text { Poids théorique } \\
\text { (en g) }\end{array}$ & $\begin{array}{c}\text { Poids observé } \\
\text { (en g) }\end{array}$ & $\begin{array}{c}\text { Moyenne observée } \\
\text { (en g) }\end{array}$ \\
\hline drachme & DR & & 6 & 3,70 & $3,04-4,08$ & 3,64 & Type \\
triobole & TR & $1 / 2$ DR & 3 & 1,85 & $1,74-1,95$ & 1,85 & DRM-11 \\
obole & OB & $1 / 6$ DR & 1 & 0,62 & $0,5-0,7$ & 0,61 & OBM-10/11 \\
grand bronze & GB & $1 / 4$ OB $\times 100$ & $1 / 4$ & 15 & $9,18-17,4$ & 13,76 & GBM-13 \\
\hline
\end{tabular}

\begin{tabular}{|l|c|c|c|c|c|c|c|}
\hline \multicolumn{1}{|c|}{$\mathbf{2 2 5 - 2 1 0}$} & \multicolumn{1}{|c|}{ Dénomination } & Abréviation & Équivalence & Rapport à l'obole & $\begin{array}{c}\text { Poids théorique } \\
\text { (en g) }\end{array}$ & $\begin{array}{c}\text { Poids observé } \\
\text { (en g) }\end{array}$ & $\begin{array}{c}\text { Moyenne observée } \\
\text { (en g) }\end{array}$ \\
\hline drachme & DR & & 6 & 3,70 & $3,04-4,08$ & 3,64 & Type \\
obole & OB & $1 / 6$ DR & 1 & 0,62 & $0,37-0,90$ & $0,57-0,63$ & OBM-10/11 \\
grand bronze & GB & $1 / 6$ OB $\times 100$ & $1 / 6$ & 10,28 & $6,17-15,21$ & $10,14-10,26$ & GBM-14/15 \\
\hline
\end{tabular}

\begin{tabular}{|c|c|c|c|c|c|c|c|}
\hline $210-90$ & & & & & & & \\
\hline Dénomination & Abréviation & Équivalence & Rapport à l'obole & $\begin{array}{l}\text { Poids théorique } \\
\text { (en g) }\end{array}$ & \begin{tabular}{|c|} 
Poids observé \\
(en g)
\end{tabular} & $\begin{array}{c}\text { Moyenne observée } \\
\text { (en g) }\end{array}$ & Type \\
\hline tétrobole & TO & & 4 & 2,60 & $1,52-3,26$ & 2,66 & DRM-16/50 \\
\hline diobole & DO & $1 / 3 \mathrm{TO}$ & 2 & 0,87 & $0,73-0,96$ & 0,84 & DOM-56/57 \\
\hline obole & OB & $1 / 4 \mathrm{TO}$ & 1 & 0,65 & $0,40-0,85$ & 0,63 & OBM-10/11 \\
\hline hémiobole & $\mathrm{HO}$ & $1 / 2 \mathrm{OB}$ & $1 / 2$ & 0,33 & $0,15-0,39$ & 0,32 & OBM-10/11 lég. \\
\hline grand bronze & GB & $1 / 8 \mathrm{OB} \times 100$ & $1 / 8$ & 8,13 & $4,08-12,54$ & $7,36-8,13$ & GBM-22/23/27 \\
\hline moyen bronze & MB & $1 / 2 \mathrm{~GB}$ & $1 / 16$ & 4,06 & $2,05-6,79$ & $4,11-4,01$ & MBM-28/33 \\
\hline petit bronze & PB & $1 / 2 \mathrm{MB}$ & $1 / 32$ & 2,03 & $1,20-3,69$ & 1,96 & PBM-29/50 \\
\hline petit bronze léger & PBL & $1 / 2 \mathrm{~PB}$ & $1 / 64$ & 1,02 & $0,80-1,34$ & 1,06 & PBM29/30 lég. \\
\hline
\end{tabular}

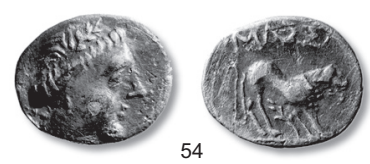

(DRM-17-9)
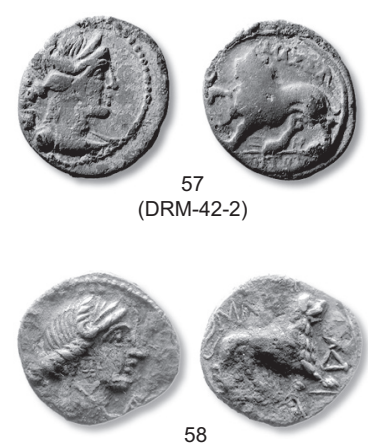

(DRM-55-24)

\begin{tabular}{|c|c|c|c|c|c|c|c|}
\hline $90-49$ & & & & & & & \\
\hline Dénomination & Abréviation & Équivalence & Rapport à l'obole & $\begin{array}{c}\text { Poids théorique } \\
\text { (en g) }\end{array}$ & \begin{tabular}{|c} 
Poids observé \\
(en g)
\end{tabular} & $\begin{array}{c}\text { Moyenne observée } \\
\text { (en g) }\end{array}$ & Type \\
\hline tétrobole & $\mathrm{PO}$ & & 4 & 2,30 & $0,85-2,93$ & 2,30 & DRM-54/56 \\
\hline obole & OB & 1/4 TO & 1 & 0,58 & $0,40-0,67$ & 0,52 & OBM-10/11 \\
\hline petit bronze & PB & $1 / 320 \times 100$ & $1 / 32$ & 1,80 & $0,71-3,40$ & 1,69 & PBM-51/68 \\
\hline très petit bronze & TPB & 1/5 PB & $1 / 160$ & 0,36 & $0,14-0,74$ & 0,39 & PBM-97/98 \\
\hline
\end{tabular}

oboles

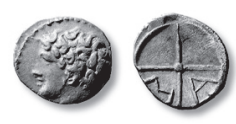

20

(OBM-10)

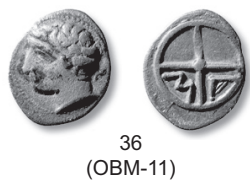

(OBM-11)

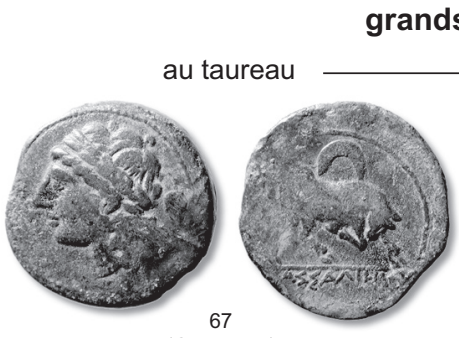

(GBM-22-7)

grands bronzes 1 au trépied

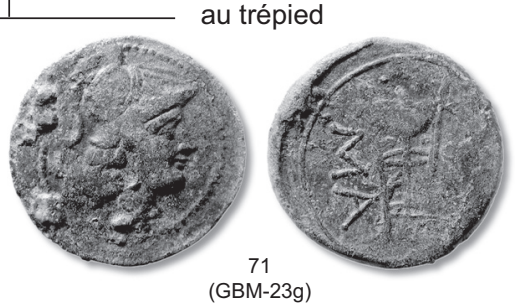

petits bronzes

moyens bronzes

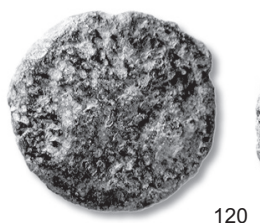

(MBM-28-10)

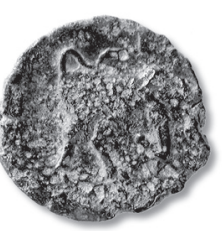

très petits bronzes

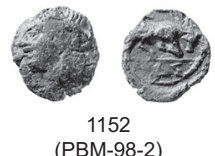

petits bronzes tardifs

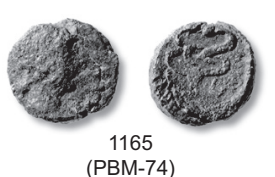

(PBM-74)

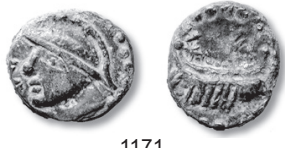

1171

(PBM-84-1)

Fig. 116 - Le système monétaire de Marseille de la fin du III ${ }^{e}$ s. au $I^{\text {er }}$ s. av. J.-C. : principaux types, métrologie et chronologie des étapes successives d'après les fouilles de Lattes et les datations archéologiques disponibles en Gaule méridionale (toutes les monnaies figurées proviennent de Lattes et sont reproduites grandeur nature) (DAO et clichés : M. Py, CNRS). 
drachmes légères (Depeyrot, 1999), joua en Gaule du Sud un rôle certainement majeur dans la propagation de l'usage des monnaies dans les sociétés autochtones. Pour autant, ce processus fut en vérité relativement lent, puisque plusieurs générations séparent son enclenchement de ses effets réels. L'analyse des contextes de découverte lattois a permis de confirmer la datation de cette réforme dans les dernières décennies du $\mathrm{III}^{\mathrm{e}} \mathrm{s}$. (fig. 116), et d'écarter définitivement la théorie développée par C. Brenot selon laquelle Marseille aurait émis une unique dénomination de bronze, progressivement dévaluée en fonction des réformes monétaires de Rome (Brenot, 1990 et 1996). Elle a montré aussi l'aspect très progressif de la diffusion de ces monnaies, qui restent rares jusqu'à la fin du $\mathrm{II}^{\mathrm{e}} \mathrm{s}$. dans le monde indigène, où l'augmentation des liquidités tient plus à l'accumulation d'espèces circulant pendant une longue période qu'à l'augmentation des frappes par le centre émetteur.

Les découvertes d'une monnaie à la croix dans un niveau de la fin du $\mathrm{III}^{\mathrm{e}} \mathrm{s}$. (Us 4475) et de sept autres dans un contexte des premières années du $\mathrm{II}^{\mathrm{e}} \mathrm{s}$. (Us 52104) sont également importantes en ce qu'elles permettent, pour la première fois, de valider par la stratigraphie l'hypothèse d'une création ancienne de ces espèces d'argent languedociennes (Soutou, 1966 ; Allen, 1969 ; Depeyrot, 2002), rendant obsolète la chronologie basse proposée par certains sur la base d'arguments historiques contestables (Richard J.-C., 1970 ; Clavel, 1970 ; Colbert de Beaulieu, 1973). La révision du trésor $n^{\circ} 3$, formé de 850 monnaies à la croix relevant toutes de types occidentaux (parmi lesquels cinq pièces à légende ibérique) et enfouies aux alentours du milieu du $\mathrm{II}^{\mathrm{e}}$ s., a également souligné les dangers que pouvait présenter la localisation des émissions en utilisant principalement les dépôts monétaires, tout en confirmant sur le plan quantitatif la persistance d'un décalage d'échelle entre thésaurisation et circulation.

Ce n'est cependant qu'à partir du dernier quart du $\mathrm{II}^{\mathrm{e}} \mathrm{s}$. que le numéraire devient assez courant dans l'habitat : Lattes ne se singularise donc pas de son environnement indigène quant à la chronologie de la monétisation des échanges internes, seule l'estimation calibrée de la quantité de monnaies en usage montrant une différence notable entre le port et son hinterland. Les données numismatiques apparaissent sur ce point en totale concordance avec les conclusions tirées de la diffusion des vaisselles et des amphores d'origine méditerranéenne, dont l'apparition est synchrone sur la côte et dans l'intérieur mais dont le volume est nettement différent; ce trait pouvant être interprété comme une conséquence indirecte de l'activité marchande du comptoir portuaire induisant un niveau de consommation supérieur à celui des zones rurales.

La situation au I ${ }^{\mathrm{er}} \mathrm{s}$. av. J.-C. est plus complexe du fait de la multiplication des frappes régionales et de l'ouverture des circuits de circulation. Ces nouvelles émissions et importations s'ajoutent aux apports de Marseille, dont la production monétaire ne faiblit pas jusqu'à sa chute devant les armées de César en 49 et dont les monnaies (désormais surtout des petits bronzes) restent largement en usage à Lattes jusqu'à l'époque augustéenne (fig. 116). Dans la partie orientale de la Gaule méditerranéenne, entre les Alpes et l'Hérault, c'est même Marseille, nonobstant la conquête militaire de la Provincia, qui sert jusqu'à l'époque triumvirale (et parfois au-delà) de référence métrologique et bien souvent iconographique pour les émissions régionales.

Ces dernières sont à Lattes principalement originaires de la région nîmoise et de la basse vallée du Rhône (y compris une importante série de potins méridionaux), tandis qu'aucun indice ne permet actuellement de caractériser d'émissions que l'on pourrait attribuer à Lattes même. Cet accrochage à l'Est reste cohérent avec l'inclusion séculaire du site dans le domaine d'influence massaliète, qui explique la modicité des apports monétaires en provenance du monde ibérique et ibéro-languedocien. Il explique aussi, parallèlement à d'autres considérations concernant l'usage spécifique du monnayage d'argent et les probables ponctions coloniales, la rareté permanente du numéraire romain, les deniers étant ici particulièrement peu fréquents.

L'importance et la chronologie des monnayages en provenance de la Gaule chevelue ont pu être discutées sur la base de la réalité objective : celle-ci ne laisse que peu de place aux vieilles théories sur l'empire arverne et sur une prétendue domination celtique sur le Midi méditerranéen, malgré les déclarations emphatiques de Strabon (Géographie, IV, 2, 3). Les statères d'or gaulois sont absents de Lattes, rares et pour la plupart tardifs ailleurs, et l'on ne saurait sur cette documentation continuer à valider la théorie générale naguère imposée à la numismatique méridionale par le $\mathrm{D}^{\mathrm{r}}$ Colbert de Beaulieu (1974). La chronologie de la diffusion des monnaies continentales, telle qu'elle ressort de la stratigraphie de Lattes, interdit également d'expliquer par la guerre des Gaules et les mouvements des troupes romaines qu'elle entraîna (Gentric, 1981), une diffusion qui apparaît plus précoce et liée principalement aux relations commerciales. Le même raisonnement peut d'ailleurs être appliqué aux quelques monnaies étrangères 
(ibériques, puniques, magno-grecques, grecques continentales et orientales) qui parviennent sur le littoral gaulois depuis les quatre coins de la Méditerranée.

La phase «protohistorique » de cette histoire numismatique se termine avec l'apparition des modules romanisés et les réformes augustéennes à l'origine de la création des dupondii de Nîmes au crocodile. Les décomptes effectués à
Lattes, rendant compte d'un affaiblissement à cette époque de la masse monétaire en circulation, ont permis de poser, à défaut de le résoudre, le problème de la déflation dont on observe la poursuite durant tout le Haut-Empire (fig. 115). L'approfondissement de cette question reviendra à l'étude de la circulation monétaire romaine sur le site de Lattes, qui reste à faire. 